\title{
Quantitative analysis of Gamma Knife stereotactic radiosurgery for uveal melanoma
}

\author{
Raj KISHOR BISHT ${ }^{1, a}$, Gopishankar NATANASABAPATHI ${ }^{2}$, Shashank SHARAD KALE ${ }^{1}$ \\ ${ }^{I}$ Department of Neurosurgery, All India Institute of Medical Sciences, New Delhi, India \\ ${ }^{2}$ Dr B.R. Ambedkar Institute Rotary Cancer Hospital, All India Institute of Medical Sciences, New Delhi, India \\ ${ }^{a}$ E-mail address: raaj_bisht@rediffmail.com
}

(received 5 December 2019; revised 19 March and 30 April 2020; accepted 4 May 2020)

\begin{abstract}
The purpose of the study was to analyze single fraction Gamma Knife stereotactic radiosurgery (SRS) for uveal melanoma (UM). In the treatment of UMs, the dose distribution exhibited by an irregular eye surface has more calculation uncertainty. A tissue-equivalent bolus was placed on the left eye surface of a human head-shaped phantom. It was assumed that the treated eye is fixed using retrobulbar anesthesia and suture on extraocular muscles for phantom study. Leksell stereotactic frame was fixed around phantom's head and the stereotactic computed tomography (CT) was performed. Two sets of scans were acquired (a) without bolus and (b) with a bolus of $1.0 \mathrm{~cm}$ thickness. These scans were transferred into a treatment planning system (TPS). The skull contouring was performed using stereotactic CT images. The target, visual pathways, and eye lens were delineated in stereotactic CT space created on TPS. A clinical relevant plan was designed on the CT study set "a" to deliver a radiation dose of 30Gy at tumor margin. The plan superimposed over CT study set "b" and compiled for convincing treatment strategy. The tumor coverage was 95\% at $50 \%$ prescription isodose line. The conformity index, selectivity and the gradient index were $1.27,0.80$ and 3.28 respectively. The left optic nerve and eye lens received a maximum dose of $11.1 \mathrm{~Gy}$ and $11.0 \mathrm{~Gy}$ respectively. The treatment plan overlay showed similar planning indices and critical organ doses. The plan comparison showed: an irradiated volume received the radiation dose $>15$ Gy varies $<1.0 \%$ whereas the volume received $<15$ Gy were larger $(>1.0 \%)$ in the study set "b". The distant lateral points from the target volume which describe the phantom's eyelid showed a radiation dose of $3.2 \mathrm{~Gy}-2.5 \mathrm{~Gy}$. The doses to these points were misled and ignored in the CT study set "a". The eye bolus provides better dosimetric information in the estimation of low dose areas which is commonly misled on TPS in SRS planning for UMs.
\end{abstract}

Key words: Gamma Knife; phantom; bolus; stereotactic radiosurgery; uveal melanoma.

\section{Introduction}

The uveal tract of the human eye consists of the ciliary body, iris, and choroid, which contains inhabitants of melanocytes. The uveal melanoma (UM) arises from melanocytes. Melanocytes reside within the stroma of the choroid (90\%), ciliary body (7\%) or the iris (2\%) [1]. UM is the most common primary intraocular malignancy in adults (mean age is 60years) with an estimated incidence of 4.9 per million men and 3.7 per million women. UM is rear but potentially life-threatening disease. These tumors originate from the skin (91\%), tissues around eye $(5 \%)$ or the mucosa (1\%) [2-6]. Ocular melanomas are likely to metastasize in the liver, breast, lung, kidney, and other regions of the body. The choroidal melanoma is the most common ocular melanoma which comprises over $75 \%$ of all intraocular melanomas [7]. Uveal melanomas are completely asymptomatic in the early stages of their development. The treatment of these tumors depends on the site, size, and location of the lesion. An extraocular extension, metastasis, and recurrence are associated with extremely poor prognosis and unexpected long term survival [7-8].

A decade before, it was believed that the treatment of metastatic melanoma is ineffective and enucleation is only and the principal treatment. In recent years, various eye-sparing treatments such as surgery, laser treatments, and specific radiotherapy techniques are popular among ophthalmologist's community. These treatment modalities replaced surgical removal techniques and grown as the most common initial therapy for small to medium-sized choroidal melanomas [917]. The local tumor control rate with these recent treatment techniques is reached more than $90 \%[9,13,15,16]$. The radiation treatment is preferable and serves an important role in organ conservation and preserve visual function. Proton beam radiotherapy (PT), plaque brachytherapy (PBT), and stereotactic radiosurgery (SRS) are the most common treatment options for UMs. Single fraction stereotactic radiosurgery with Gamma Knife (GK) is an imperative choice in the treatment of patients with malignant melanoma of the ciliary body. 
In this study, the Gamma Knife stereotactic radiosurgery (GKSRS) of uveal melanoma is presented using a human headshaped phantom [18]. The Leksell Gamma Plan (Elekta AB, Stockholm, Sweden) Software Version 10.1 (LGP) treatment planning system for GK exhibits more uncertainty in dose distribution while planning of peripheral targets. This may be due to imprecise modeling in the buildup region. The displayed dose distribution may mislead planning indices and certainly incapable to explain low dose areas for such cases. In this study, a strategy of using easily available, economical, and patient-friendly bolus material is presented to improve surface irregularities and quantify lower dose areas in the radiation field. We described an inevitability of beam modifier in GKSRS technique to treat intraocular tumors. To the best of our knowledge, this is the first report on using bolus at the irregular surface of the eye to improve the dose distribution pattern in the treatment planning system.

\section{Material and methods}

\section{The phantom study}

An in-house made human head shaped phantom was utilized to evaluate and analyze single-fraction radiosurgery for UMs [18]. The phantom is made of poly methyl methacrylate (PMMA), commercially known as perspex or acrylic material. It was believed that the eye movement is well controlled using anesthesia and sutures for phantom studies. The Leksell stereotactic frame was fixed around the head of the phantom using four pin fixation posts. The head phantom was positioned on the couch of computed tomography (CT) scanner with CT indicator box to acquire stereotactic images. The stereotactic CT imaging was done using Siemens Emotion 6 CT scanner (Siemens Healthcare, Erlangen Germany). The left eye of the phantom was roofed with a tissue-equivalent bolus sheet for the treatment and plan evaluation (Figure 1). The density of the bolus is $1.03 \mathrm{~g} / \mathrm{cc}$. The TMR10 dose algorithm in LGP version 10.1 treatment planning system enables calculation of dose at arbitrary points in the head. It models all tissue in the head as water and referred to as a "water-based" algorithm. The bolus is commercially available and often used in radiotherapy departments. The total thickness of the bolus was kept $10 \mathrm{~mm}$ in view of analyzing lower dose areas. A metal marker was placed on the surface of the phantom's nose (point $\mathrm{P}$, Figure 2) to locate and measure radiation dose at arbitrarily chosen points. The location of these points refers to the radiation dose on the phantom's eyelid (Figure 3a \& b). Two sets of CT scan were acquired; (a) without bolus and (b) phantom's eye roofed using bolus. The scanning parameters were as follows: tube voltage $120 \mathrm{KVp}$, Tube current $140 \mathrm{~mA}$, slice thickness $1.0 \mathrm{~mm}$, and FOV $230 \times 230 \mathrm{~mm}^{2}$. The stereotactic CT images of the phantom were imported into LGP and defined to create routine three-dimensional modeling. The maximum fiducial defining error in creating stereotactic space for treatment planning was $0.9 \mathrm{~mm}$.

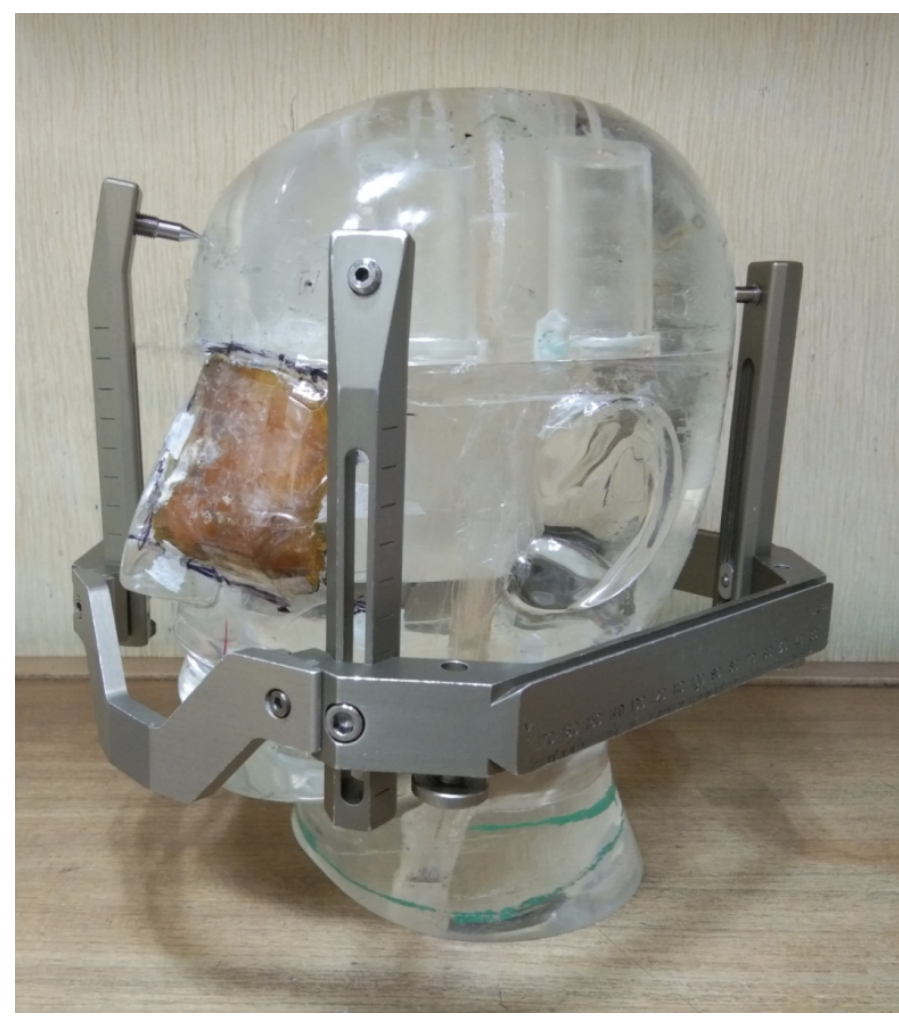

Figure 1. Leksell frame fixation and application of tissueequivalent bolus on the left eye of the head phantom

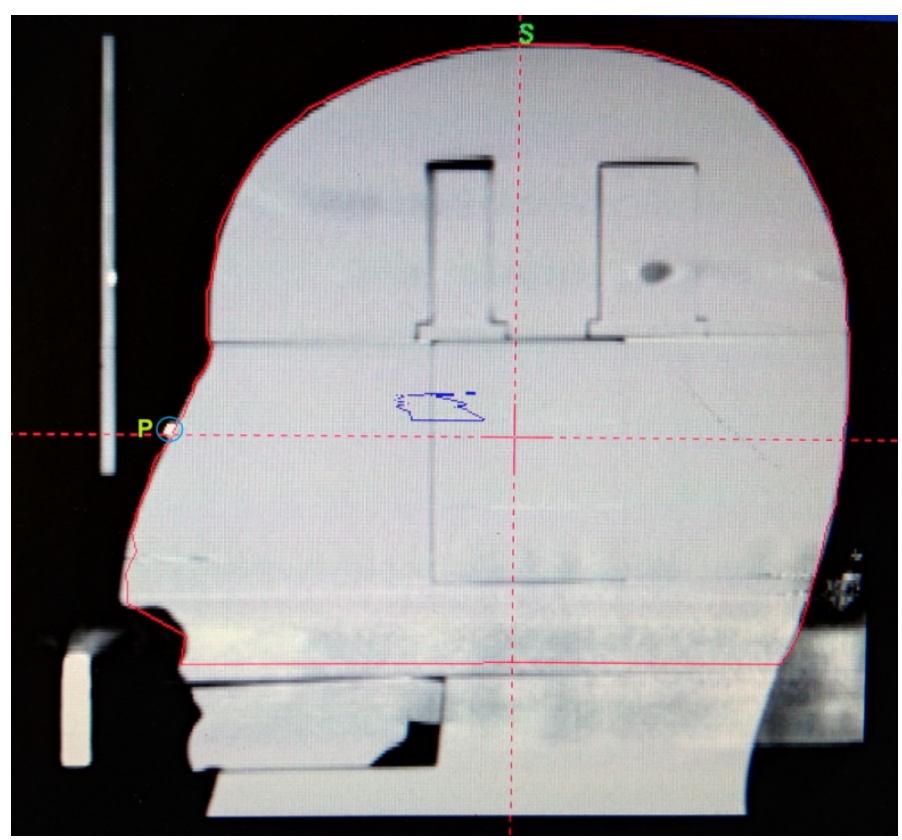

Figure 2. Metal marker and location of point "P" 


\section{Treatment planning}

A clinically relevant plan for the treatment of uveal melanomas was designed on LGP using acquired stereotactic CT images of the phantom. The CT images were utilized to contour the outer surface of the phantom head. The target, eye lens and optic nerves were delineated on axial CT images. A single-fraction stereotactic radiosurgery treatment plan was prepared using various planning tools on the first set of CT image sequence "a". The target matrix grid size of $2.0 \mathrm{~mm}$ was preferred to analyze major planning indices and lower radiation dose areas. An Inverse planning strategy on LGP 10.1 was utilized to prepare a precise treatment plan. Dynamic shaping, manual blocking of collimator sectors, collimation pattern and isocenter adjustment of practical shots were attempted to limit radiation doses to the nearby structures and improve planning indices.

The second set of CT image sequence "b", was transferred into the treatment planning system. An effort was made to define CT sequence with similar fiducial position error in image planes (mean $0.5 \mathrm{~mm}$, $\max 0.9 \mathrm{~mm}$ ) to create equivalent stereotactic space similar to study "a". The delineated volumes and prepared treatment plan were overlayed on the second image sequence. The planning indices and preferred lower dose regions for both treatment approaches were evaluated.

\section{Results}

\section{Phantom data}

The delineated target volume for the phantom study was $1.91 \mathrm{cc}$. A marginal dose of $30 \mathrm{~Gy}$ was delivered to the target volume at $50 \%$ prescription isodose line. The treatment plan consists of 4 isocenters of composite collimator shots. Among planned shots, two shots required dynamic shaping to reduce doses to the left eye lens and optic nerve. The prescription isodose volume (PIV) was able to envelop $95 \%\left(1.82 \mathrm{~cm}^{3}\right)$ of the tumor volume. The maximum point dose of $61.2 \mathrm{~Gy}$ $(\sim 102 \%)$ and the minimum dose of $21.3 \mathrm{~Gy}(\sim 35.5 \%)$ was recorded within the target volume. The conformity index $(\mathrm{CI})$, selectivity (S) and gradient index (GI) were calculated as 1.27, 0.80 and 3.28 for the prepared treatment plan (Table 1). The volumes of the left optic nerve and right optic nerve were $1.11 \mathrm{~cm}^{3}$ and $1.21 \mathrm{~cm}^{3}$ for the phantom. The maximum point doses delivered to the left optic nerve and left eye lens were recorded as 11.1 Gy and 11.0 Gy respectively (Table 2).

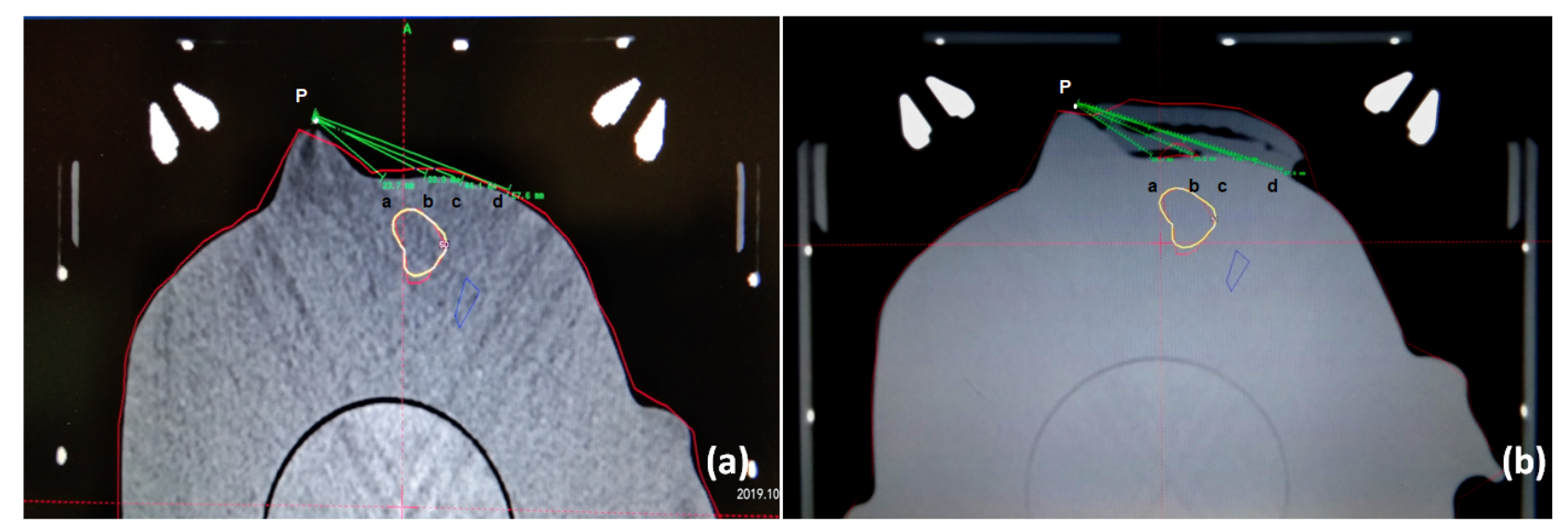

Figure 3. An investigation of left eyelid dose at points a, b, c and d; (a) without bolus and (b) with bolus
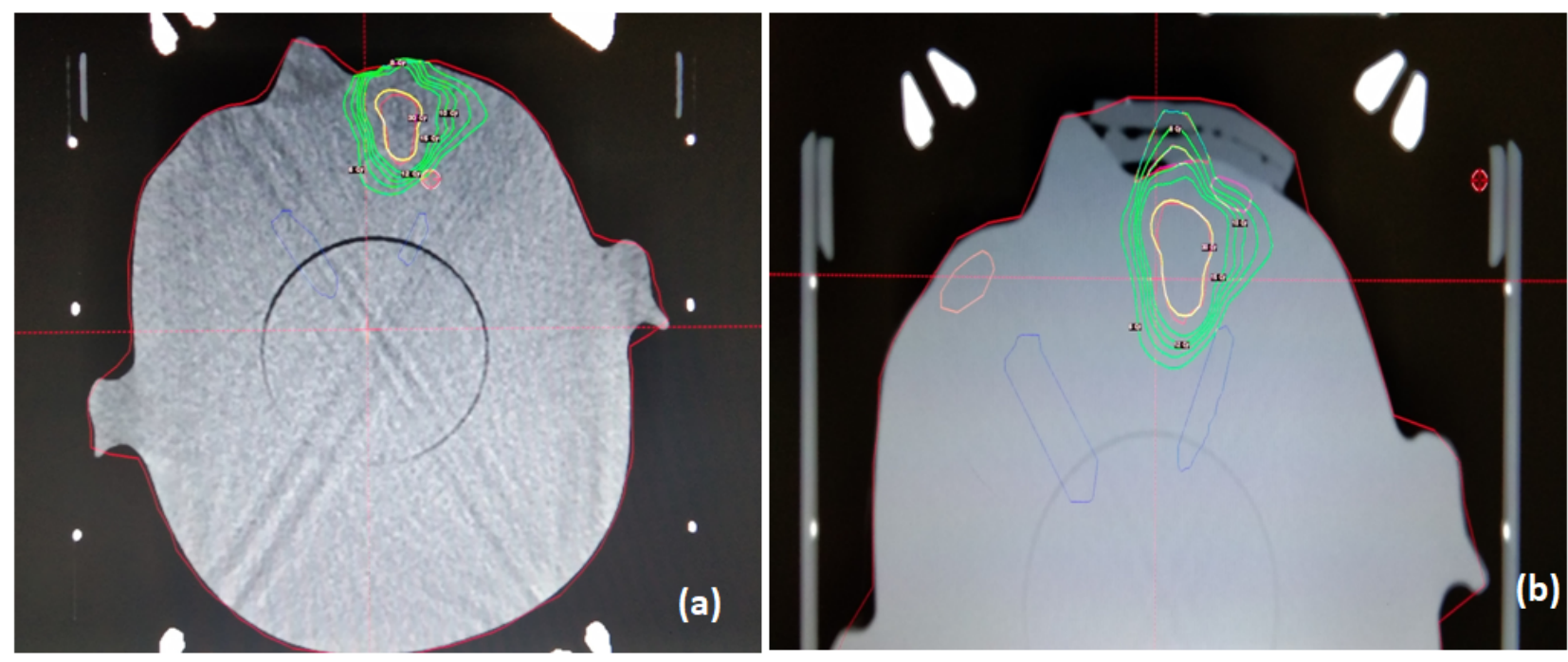

Figure 4. Displayed isodose curves (a) without bolus and (b) with bolus study 
In the treatment plan overlay, the phantom surface was recontoured using CT images with intact eye bolus roofed over irregular left eye surface of the phantom. The planning parameters were analyzed on the treatment planning system (Table $1 \&$ Table 2). No significant variation has been observed in tumor coverage, prescription isodose volume, conformity index, selectivity and gradient index on comparison however, the total treatment time increased by $3.1 \mathrm{~min}$ for roofed bolus study (Table 1).

Table 3, illustrates a comparative study for higher and lower radiation dose volumes. For the doses $\geq 15 \mathrm{~Gy}$, the variation in the dose volumes were $<0.96 \%$, whereas the variation increases for the radiation doses $<15$ Gy gradually. The bolus also shares a significant fraction of dose volume in an evaluation of lower dose volumes, however, the volume received lower doses within the phantom anatomy was also larger in bolus study.

The radiation dose delivered to the left eyelid was also analyzed in both treatment conditions. Four distinct points; a, b, c \& d on the outer surface of the phantom eyelid were chosen and analyzed for either treatment condition (Figure 3a \& b, Table 4).

Table 1. Treatment and quality parameters of designed plan

\begin{tabular}{|c|c|c|c|c|}
\hline S No & & Without bolus & With bolus & Variation \\
\hline 1 & Maximum dose to the tumor volume & $61.2 \mathrm{~Gy}$ & $61.1 \mathrm{~Gy}$ & Non significant \\
\hline 2 & Minimum dose to the tumor volume & $21.3 \mathrm{~Gy}$ & 21.8 & $2.29 \%$ \\
\hline 3 & Mean Dose to the tumor volume & $41.0+6.9 \mathrm{~Gy}$ & $40.9+6.8 \mathrm{~Gy}$ & Non significant \\
\hline 4 & Tumor volume within PIV & $1.82 \mathrm{~cm}^{3}$ & $1.82 \mathrm{~cm}^{3}$ & Non significant \\
\hline 5 & PIV Matrix & $2.22 \mathrm{~cm}^{3}$ & $2.21 \mathrm{~cm}^{3}$ & Non significant \\
\hline 6 & $\mathrm{CI}_{\mathrm{RTOG}}$ & 1.27 & 1.28 & Non significant \\
\hline 6 & CI (Paddick) & 0.781 & 0.784 & Non significant \\
\hline 7 & Selectivity & 0.80 & 0.81 & Non significant \\
\hline 8 & Gradient Index & 3.28 & 3.27 & Non significant \\
\hline 9 & Treatment time & $107.1 \mathrm{~min}$ & $110.2 \mathrm{~min}$ & $3.1 \mathrm{~min}$ \\
\hline 10 & Integral dose to the tumor volume & $78.3 \mathrm{~mJ}$ & $78.3 \mathrm{~mJ}$ & Non significant \\
\hline 11 & Skull Volume & $3811.18 \mathrm{~cm}^{3}$ & $3834.74 \mathrm{~cm}^{3}$ & Non significant \\
\hline
\end{tabular}

Table 2. Radiation dose to the critical structures (the radiation doses were displayed by the LGP 10.1 treatment planning system and not measured by any other tools)

\begin{tabular}{|c|c|c|c|c|}
\hline \multirow[b]{2}{*}{ Critical Organ } & \multicolumn{2}{|c|}{ Plan (a) Without bolus } & \multicolumn{2}{|c|}{ Plan (b) With bolus } \\
\hline & Max Dose (Gy) & Mean Dose (Gy) & Max Dose (Gy) & $\begin{array}{c}\text { Mean Dose } \\
\text { (Gy) }\end{array}$ \\
\hline Optic nerve Left (Volume $1.11 \mathrm{~cm}^{3}$ ) & 11.0 & $3.2 \pm 1.7$ & 11.1 & $3.1 \pm 1.7$ \\
\hline Optic nerve Right (Volume $1.21 \mathrm{~cm}^{3}$ ) & 1.6 & $0.8 \pm 0.2$ & 1.7 & $0.8 \pm 0.2$ \\
\hline Eye Lens Left (Volume 196.9mm³) & 11.7 & $6.9 \pm 1.6$ & 11.9 & $6.9 \pm 1.6$ \\
\hline Eye Lens Right (Volume $200.2 \mathrm{~mm}^{3}$ ) & 0.7 & $0.4 \pm 0.1$ & 0.7 & $0.4 \pm 0.1$ \\
\hline
\end{tabular}

Table 3. Dose volumes, where $V_{90 \%}, V_{80 \%}, V_{70 \%} V_{50 \%}$ are the target matrix volumes enclosed with respective isodose curve and $V_{20 G y}$, $V_{15 G y}$ $V_{10 G y} V_{8 G y}$ and $V_{6 G y}$ are the target matrix volume received corresponding radiation dose

\begin{tabular}{|c|c|c|c|c|c|c|}
\hline S No & $\begin{array}{l}\text { Relative and } \\
\text { absolute dose volume }\end{array}$ & Without bolus (A) & With bolus (B) & $\%$ Variation & $\begin{array}{l}\text { The bolus volume contribution for } \\
\text { respective isodose curve }(C)\end{array}$ & $\begin{array}{l}\text { Variation in dose volume } \\
\text { within phantom anatomy } \\
\text { (B-C-A) }\end{array}$ \\
\hline 1 & $\mathrm{~V}_{90 \%}$ & $21.7 \mathrm{~mm}^{3}$ & $21.6 \mathrm{~mm}^{3}$ & -0.46 & 0 & Within phantom anatomy \\
\hline 2 & $\mathrm{~V}_{80 \%}$ & $247.8 \mathrm{~mm}^{3}$ & $246.1 \mathrm{~mm}^{3}$ & -0.68 & 0 & Within phantom anatomy \\
\hline 3 & $\mathrm{~V}_{70 \%}$ & $771.2 \mathrm{~mm}^{3}$ & $765.2 \mathrm{~mm}^{3}$ & -0.77 & 0 & Within phantom anatomy \\
\hline 4 & $\mathrm{~V}_{50 \%}$ & $2.26 \mathrm{~cm}^{3}$ & $2.24 \mathrm{~cm}^{3}$ & -0.88 & 0 & Within phantom anatomy \\
\hline 5 & $\mathrm{~V}_{20 \mathrm{~Gy}}$ & $4.64 \mathrm{~cm}^{3}$ & $4.60 \mathrm{~cm}^{3}$ & -0.86 & 0 & Within phantom anatomy \\
\hline 6 & $\mathrm{~V}_{15 \mathrm{~Gy}}$ & $7.26 \mathrm{~cm}^{3}$ & $7.19 \mathrm{~cm}^{3}$ & -0.96 & 0 & Within phantom anatomy \\
\hline 7 & $\mathrm{~V}_{12 \mathrm{~Gy}}$ & $9.87 \mathrm{~cm}^{3}$ & $10.66 \mathrm{~cm}^{3}$ & +1.8 & $0.599 \mathrm{~cm}^{3}$ & $191 \mathrm{~mm}^{3}$ \\
\hline 8 & $V_{10 G y}$ & $12.50 \mathrm{~cm}^{3}$ & $13.31 \mathrm{~cm}^{3}$ & +6.08 & $0.654 \mathrm{~cm}^{3}$ & $156 \mathrm{~mm}^{3}$ \\
\hline 9 & $\mathrm{~V}_{8 \mathrm{~Gy}}$ & $16.70 \mathrm{~cm}^{3}$ & $18.54 \mathrm{~cm}^{3}$ & +9.92 & $1.332 \mathrm{~cm}^{3}$ & $508 \mathrm{~mm}^{3}$ \\
\hline 10 & $\mathrm{~V}_{6 \mathrm{~Gy}}$ & $24.39 \mathrm{~cm}^{3}$ & $27.73 \mathrm{~cm}^{3}$ & +12.04 & $3.030 \mathrm{~cm}^{3}$ & $310 \mathrm{~mm}^{3}$ \\
\hline 11 & $\mathrm{~V}_{9.5 \mathrm{~Gy}}$ & $13.41 \mathrm{~cm}^{3}$ & $14.66 \mathrm{~cm}^{3}$ & +7.29 & $0.999 \mathrm{~cm}^{3}$ & $251 \mathrm{~mm}^{3}$ \\
\hline
\end{tabular}


Table 4. Doses at arbitrarily chosen points on eyelid of the phantom (doses were displayed by the LGP 10.1 treatment planning system and not measured by any other tools)

\begin{tabular}{|c|c|c|}
\hline $\begin{array}{l}\text { Points on phantom eye surface } \\
\text { (distance from Point " } P \text { ") }\end{array}$ & Point dose with eye bolus (Gy) & Point dose without eye bolus (Gy) \\
\hline Point a $23.7 \mathrm{~mm}$ & 4.0 & 6.3 \\
\hline Point b $33.6 \mathrm{~mm}$ & 5.8 & 6.0 \\
\hline Point c $44.1 \mathrm{~mm}$ & 3.2 & 0.0 \\
\hline Point d $57.6 \mathrm{~mm}$ & 2.5 & 0.0 \\
\hline
\end{tabular}

\section{Discussion}

Small uveal melanomas are usually treated with transpupillary thermotherapy or PBT, however, large UMs are still a therapeutic challenge. Tumor resection combined with radiation therapy is another eye preserving management has been opted by various researchers $[14,15]$. Radiotherapy alone for larger tumor with high dose seems to be associated with increased long term complications including toxic tumor syndrome or persistent exudative retinal detachment leading to visual loss or enucleation [16,17]. PBT therapy is the most common treatment for choroidal melanoma. Various isotopes have been used for PBT. The most common are gammaemitting radionuclides such as Iodine-125, Cesium-131, Palladium-103. The Ruthenium-106, which emits $\beta$-particles, can be used however its limited tissue penetration of $4 \mathrm{~mm}=$ $5 \mathrm{~mm}$ makes it suitable only for tumors less than $5 \mathrm{~mm}$ in height [19]. Results of overall survival, visual outcome, and local tumor control, in patients who received stereotactic radiation appear equivalent to those undergoing plaque radiotherapy [10,20].

The radiation therapy with Cyber Knife, linear accelerators, charge particles and GK has been used successfully in the management of choroidal melanoma. Unlike PBT, which requires two procedures on separate dates - placing and removing a plaque, GKSRS is a same-day procedure. The PT facilities are expensive, resource intensive, and not commonly available.

The perspex phantoms are commonly used in radiotherapy departments for routine quality assurance tests and treatment verifications of common to complex patient-specific treatments. A human head-shaped phantom was used to validate the perception of the present study. Tissue equivalent bolus is often used in radiotherapy when treating uneven areas of the patient's surface or to provide dose build-up to the skin surface as they are very flexible. In the present study, the bolus was used to make up the missing tissue near eye region. Establishing stereotactic space using bolus to level irregular eye gives realistic dosimetric information on calculating planning indices.

The treatment plan overlay showed close agreement in the calculation of major planning indices. It was observed that the integral curve of "the half prescription isodose line (PIV25\%)" was within the phantom anatomy, which supports identical gradient index for both treatment policies. The volume enclosed with the radiation doses > 15 Gy (PIV25\%) are practically similar for both studies. These dose volume segments are inside the tumor volume and barely contribute any clinical significance. Further analysis of dose volumes $<15$ Gy illustrates that size of composite volumes is larger in bolus based calculations. Since the bolus body shares a volume fraction for lower isodose volumes, these part volumes were drawn on TPS for relevant evaluation. It was observed that the dose distribution and variation in lower dose volumes were neatly appreciated in bolus study (Figure 4). For example; the 6 Gy dose volume difference was $3.34 \mathrm{~cm}^{3}$ (Table 3) and the volume delineated in the bolus sheet for $6 \mathrm{~Gy}$ volume was $3.03 \mathrm{~cm}^{3}$. The comparison showed $27.73 \mathrm{~cm}^{3}-3.03 \mathrm{~cm}^{3}=$ $24.70 \mathrm{~cm}^{3}$ volume received a radiation dose of $6 \mathrm{~Gy}$ inside the phantom, which is nearly $310 \mathrm{~mm}^{3}$ more than the experiment performed without bolus. The result is unambiguous and dosimetrically realistic in treating UMs and probably for other peripheral targets.

The entire dose distribution displayed on TPS lacks in realistic calculation, if the lesion is large and located along of left/right temporal globe. These cases evidently show uncertain dose distribution for lower to higher isodose regions on the patient surface. An ambiguity in enclosed volumes certainly misleads in calculating major planning indices like PIV, S, CI and others. The study obviously postulates that it is impossible to accurately calculate the lower dose volumes without placing bolus or alternative tissue-equivalent sheets on the eye surface in the treatment of UMs and/or other peripheral tumors. In the treatment of UMs, steep isodose gradients on the patient surface mislead the TPS calculations.

In single-fraction stereotactic radiosurgery with GK, skull geometry generally is defined by a dedicated scalar measurement bubble tool. Considering peripheral tumor below the irregular surface, the head surface of the phantom was generated on TPS using CT images in conjunction with the Leksell skull frame. Additionally, the skull model created from CT images reduced the dosimetric uncertainty caused by different skull measurers [21]. LGP tools were used to improve and smooth CT based phantom head contouring for experiments. In the planning strategy for UMs, the stereotactic CT scan accounts for the eyelid contouring which may overlook in the case of manual contouring using the scalar measurement bubble. Table 4 shows, though the dose received on the surface of the eyelid near target were similar in either treatment policies however the dose to the points i.e. few $\mathrm{mm}$ 
off-field, TPS absolutely misinform the absorbed dose. The radiation dose at "point c" and "point d" was evaluated as $3.2 \mathrm{~Gy}$ and $2.5 \mathrm{~Gy}$ in bolus study whereas the original treatment plan showed no dose at these points. The eye bolus of larger thickness can be used to evaluate further lower dose volumes to validate GKSRS for UMs and other peripheral targets. The present study is preliminary and indicative to check the distribution of the doses of therapeutic significance. Since the study is a dosimetric comparison on the treatment planning system for ready reference, an extension of study and comparison using films, polymer gels and other external dosimeters are further scope of verification and validation of GKSRS for UMs and other peripheral targets.

Radiotherapy for uveal melanoma is generally regarded as a reasonable treatment option. Most patients prefer eye-salvaging methods to protect the globe and ultimate vision. However, the problem of every conservative treatment is long-term visual acuity loss. PBT and PT are preferable radiation treatment in the management of melanoma. GKSRS delivers a cumulative single-day dose equivalent to 5 days of PBT or PT. Short treatment time and treatment precision with little collateral damage is a major benefit of choosing GKSRS.

\section{Conclusions}

Single fraction stereotactic radiosurgery using GK is safe and a non-invasive procedure for intraocular tumor. Modification in the patient's surface using tissue-equivalent bolus is easy, economical and comfortable to the patient in the treatment of uveal melanoma. Smoothening lower isodose lines outside the patient surface assist in calculating quality indices accurately for peripheral tumors. The bolus of suitable thickness provides adequate thickness around the patient surface and helps in the estimation of dose distribution at lower dose regions correctly, which is generally mislead by the treatment planning system for peripheral tumors. We have demonstrated the role of bolus in estimating accurate dose delivery for UMs. Combined institutional clinical results and the planning strategies would motivate to further improve the method and dose delivery regimen for GKSRS of UMs and other peripheral targets.

\section{Acknowledgements}

This study was partially supported by an intramural research project of "All India Institute of Medical Sciences" New Delhi, India [Project No A-247 at Gamma Knife Unit, Department of Neurosurgery Neurosciences centre].

\section{References}

[1] Shields CL, Kaliki S, Furuta M, et al. Clinical spectrum and prognosis of uveal melanoma based on age at presentation in 8,033 cases. Retina. 2012;32(7):1363-1372.

[2] Chang AE, Karnell LH, Menck HR. The National Cancer Data Base report on cutaneous and noncutaneous melanoma: a summary of 84,836 cases from the past decade. The American College of Surgeons Commission on Cancer and the American Cancer Society. Cancer. 1998;83(8):1664-1678.

[3] Gunduz K, Bechrakis NE. Exoresection and endoresection for uveal melanoma. Middle East Afr J Ophthalmol. 2010;17(3):210-216.

[4] Virgili G, Gatta G, Ciccolallo L, et al. Incidence of uveal melanoma in Europe. Ophthalmology. 2007;114(12):2309-2315.

[5] Isager P, Osterlind A, Engholm G, Heegaard S, Lindegaard J, Overgaard J, Storm HH. Uveal and conjunctival malignant melanoma in Denmark, 1943-97: incidence and validation study. Ophthalmic Epidemiol. 2005;12(4):223-232.

[6] Singh AD, Topham A. Incidence of uveal melanoma in the United States: 1973-1997. Ophthalmology. 2003;110:956-961.

[7] Furdova A, Strmen P, Sramka M. Complications in patients with uveal melanoma after stereotactic radiosurgery and brachytherapy. Bratisl Lek Listy. 2005;106(12):401-406.

[8] Laver NV, McLaughlin ME, Duker JS. Ocular melanoma. Archives of pathology \& laboratory medicine. 2010;134(12):1778-1784

[9] Karkhaneh R, Chams H, Amoli FA, et.al. Long-term surgical outcome of posterior choroidal melanoma treated by endore section. Retina.2007;27(7):908-914.

[10] Dunavoelgyi R, Dieckmann K, Gleiss A et al. Local tumor control, visual acuity and survival after hypofractionated stereotactic photon radiotherapy of choroidal melanomain 212 patients treated between 1997 and 2007. Int J Radiat Oncol Biol Phys. 2011;81(1):199-205.

[11] Desjardins L, Lumbroso-Le Rouic L, Levy-Gabriel C, et al. Treatment of uveal melanoma by accelerated proton beam. Dev Ophthalmol. 2012;49:41-57.

[12] Marconi DG, de Castro DG, Rebouças LM, et al. Tumor control, eye preservation and visual outcomes of ruthenium plaque brachy therapy for choroidal melanoma. Brachytherapy. 2013;12(3):235-259.

[13] Correa R, Pera J, Gómez J, et al.125I episcleral plaque brachytherapy in the treatment of choroidal melanoma: a single-institution experience in Spain. Brachytherapy. 2009;8(3):290-296.

[14] Verschueren KM, Creutzberg CL, Schalij-Delfos NE, et al. Long-term outcomes of eye-conserving treatment with ruthenium106 brachytherapy for choroidal melanoma. Radiother Oncol. 2010;95(3):332-338. 
[15] Chojniak MM, Chojniak R, NishimotoI N, et al. Primary transpupillary thermotherapy for small choroidal melanoma. Graefes Arch Clin Exp Ophthalmol. 2011;249(12):1859-1865.

[16] Robertson DM. Melanoma endoresection: aperspective. Retina.2001;21(5):403-407.

[17] Bechrakis NE, Petousis V, Willerding G et al. Ten-year results of transscleral resection of large uveal melanomas: local tumour control and metastatic rate. Br J Ophthalmol. 2010;94(4):460-466.

[18] Bisht RK, Kale SS, Natanasabapathi G, et al. Verification of Gamma Knife based fractionated radiosurgery with newly developed head-thorax phantom. Radiat Measur. 2016;91:65-74. doi: 10.1016/j.radmeas.2016.06.001.

[19] Wilkinson DA, Kolar M, Fleming PA, Singh AD. Dosimetric comparison of 106Ru and 125I plaques for treatment of shallow $(<$ or $=$ $5 \mathrm{~mm}$ ) choroidal melanoma lesions. Br J Radiol. 2008;81(970):784-789.

[20] Wackernagel W, Holl E, Tarmann L, et al. Local tumour control and eye preservation after gamma-knife radiosurgery of choroidal melanomas. Br J Ophthalmol. 2014;98:218-223.

[21] Nakazawa H, Komori M, Mori Y, et al. Effect of skull contours on dose calculations in Gamma Knife Perfexion stereotactic radiosurgery. J Appl Clin Med Phys. 2014;15(2):4603. doi:10.1120/jacmp.v15i2.4603 